Instructions for authors, subscriptions and further details:

http://brac.hipatiapress.com

\title{
Génesis del Arte Minimalista: Aportación Mexicana
}

\section{Pablo Estévez Kubli ${ }^{1}$}

1) Facultad de Artes y Diseño. Universidad Nacional Autónoma de México. México

Date of publication: June $3^{\text {rd }}, 2014$

Edition period: February 2014-June 2014

To cite this article: Estévez, P. (2014). Génesis del arte minimalista: Aportación mexicana. Barcelona, Research, Art, Creation, 2(2), 201-220. doi: 10.4471/brac.2014.09

To link this article: http://dx.doi.org/10.4471/brac.2014.09

\section{PLEASE SCROLL DOWN FOR ARTICLE}

The terms and conditions of use, except where otherwise noted, are related to the Open Journal System and to Creative Commons Attribution License (CCBY). The indication must be expressly stated when necessary. 


\title{
Minimal Art Genesis: Mexican Contribution
}

Pablo Estévez Kubli

National Autonomous University of Mexico

(Received: 20 January 2014; Accepted: 30 April 2014; Published: 3 June 2014)

\begin{abstract}
The article addresses the minimalist proposals of Mathias Goeritz and Luis Barragán from 1953, the Mexicans precursors of architectural and sculpture objects contributed to sustain the minimal art avant-garde. American artists of the sixties systematized in part the findings of Mexican architects in implementing minimum structures. The text proposes correspondences plastic root of Mexicans and Americans sculptors, using the method of comparative analysis. Furthermore, the Americans artist's sculptural theories and systems of construction to developed for the trend. Also, it is verified that the Mexican minimalist production incorporated formal compositional elements developed by the Americans. The text states that the Mexican minimalist work was consolidated in parallel with the Americans, with photographs and references substantiating examples of equivalent creations that enrich the avant-garde. Finally, we address the contemporary minimalist assemblage.
\end{abstract}

Keywords: Genesis, minimalism, correspondence, analysis, planimetric, assemblage, Mexican precursors, objects, parameters, contemporary, minimal art. 


\section{Génesis del Arte Minimalista: Aportación Mexicana}

Pablo Estévez Kubli

Universidad Nacional Autónoma de México

(Recibido: 20 Enero 2014; Aceptado: 30 Abril 2014; Publicado: 3 Junio 2014)

\section{Resumen}

El artículo aborda las propuestas minimalistas de Mathias Goeritz y Luis Barragán, a partir de 1953; los precursores mexicanos aportaron obra escultórica y arquitectónica para sustentar la vanguardia del minimal art. Los artistas estadounidenses de los años sesenta sistematizaron en parte los hallazgos de los arquitectos mexicanos en la ejecución de sus estructuras mínimas. El texto propone correspondencias plásticas de norteamericanos y mexicanos de raíz minimalista, utilizando el método de análisis comparativo. Por otro lado, los artistas estadunidenses desarrollaron teorías y sistemas de construcción para la tendencia. Así también, se verifica que la producción minimalista mexicana incorporó elementos formales compositivos desarrollados por los norteamericanos. En el texto se afirma que la obra minimalista mexicana se consolidó de forma paralela a la norteamericana, ejemplificándose con fotografías y referencias probatorias de creaciones equivalentes que enriquecen la vanguardia. Por último, se plantea la vigencia del ensamblaje minimalista contemporáneo.

Palabras clave: Génesis, minimalismo, correspondencia, planimétrico, análisis, ensamblaje, precursores mexicanos, objetos, parámetros, contemporaneidad, minimal art. 
1 origen de la vanguardia denominada minimal art corresponde a una cadena de acontecimientos y acciones que condujeron a un resultado concreto, incidiendo con propuestas plásticas en el siglo pasado. En tal sentido, Barbara Rose en su artículo $A B C A r t^{1}$ esgrimió que "la génesis del minimal como una reacción contra la subjetividad y los excesos del expresionismo abstracto" (Guasch, 2009, p. 158). El objetivo del artículo consiste en presentar concatenaciones de hechos artísticos que labraron el minimalismo en ambos países. Los inicios de la tendencia de la estructura mínima surgen en 1953 en México, con edificaciones arquitectónicas y esculturas urbanas de Mathias Goeritz y Luis Barragán. La literatura del minimalismo carece de referencias contundentes de nuestros artistas nacionales que provocaron el minimalismo. Encontramos que en la mayoría de los libros, ensayos y artículos sobre el minimal art se refieren a la aportación norteamericana como arranque de la vanguardia sin incorporar a la discusión la obra de Barragán y Goeritz. Por otro lado, consideramos que la obra escultórica minimalista realizada en México contiene diversas influencias formales, correspondencias compositivas y referencias conceptuales de la metodología estadounidense de los años sesenta del siglo pasado.

Sin embargo, los productores nacionales en la década de los setenta realizaron esculturas con una epidermis geométrica mínima, con formas cerradas y con la encomienda de emplazarlas en espacios públicos. Así también, los escultores nacionales en paralelo desarrollaron el concepto de la planimetría consistente en la aplicación de laminados metálicos con intercalación de vacíos; siguiendo de manera parcial las teorías de los minimalistas norteamericanos. Por otro lado, la literatura nos señala que Richard Wollheim en 1965 acuño el término "arte minimalista" (Schneckenburger, Ruhrberg, Frickey y Honnef, 2001, p. 524).

El minimal art consagra las estructuras de fachada industrial sustentadas en figuras geométricas, sistema modular, secuencia de partes, materiales laminados en emplazamientos en galerías, museos y espacios públicos. La investigación confirma similitudes, contrastes y correspondencias como la aplicación de sistemas industriales con materiales de la modernidad maquinista $\mathrm{y}$, sobre todo, la propuesta de formas abstractas-geométricas, composiciones formales y actitudes conceptuales de los escultores en su contexto. Así también, los minimalistas norteamericanos como Donald Judd y Robert Morris desarrollaron textos críticos que fundamentan la tendencia. 
Consideramos que en México los escultores de la década de los setenta construyeron las bases artísticas para que surgieran estructuras locales, asimilando los principios del minimal art. La aplicación inicial de la tendencia dificultaba establecer un orden minimalista en el quehacer de los escultores nacionales, ya que interpretaron con su lenguaje los parámetros vanguardistas conforme a conceptos y temáticas personales. Así también, utilizaron materiales industriales como laminados en metal, plástico y madera; construyendo estructuras con el sistema de maquila en talleres especializados al igual que sus colegas norteamericanos.

La incorporación del minimalismo emerge de manera amplia en la década de los setenta. El auge se inclina al final del siglo, con esculturas monumentales como La Puerta de Monterrey (1985) y Cabeza de caballo (1992), ambas del escultor Sebastián. Consolidamos la tendencia en los años noventa con varios escultores como Jesús Mayagoitia y Manuel Felguérez con propuestas de obra pública geométrica. En la Ciudad de México se construyeron la mayoría de las obras emblemáticas, marcando la diferencia entre lo escultórico tradicional y las piezas de arte minimalista en el país. El surgimiento de la tendencia con esculturas de bloque sellado generó un desconcierto entre la comunidad artística mexicana, al sustituir la tradición del desbaste, talla y modelado con láminas soldadas en estructuras mínimas. En tal sentido, constatamos que la línea de investigación nacional corresponde a influencias de objetos minimalistas de los años sesenta y sobre todo, por el antecedente de las obras de Goeritz y Barragán. En tal sentido, la aplicación de los parámetros minimalistas en nuestro país en la obra pública geométrica, refrendó en su momento la entrada a la modernidad escultórica (1985-1992) $\mathrm{y}$, además ampliamos el género con obra posminimalista en el presente siglo.

Así también, es necesario divulgar la aportación mexicana de tendencia minimalista fundamentada en autores relevantes que aludan a la génesis nacional. Además, analizaremos las propuestas estadounidenses y mexicanas con referencias plásticas para determinar la vinculación entre escultores de ambos países. El objetivo del artículo es evidenciar similitudes e influencias del minimal art entre productores y proponer un contenido formal para su entendimiento y valoración, a partir de las obras de los artistas nacionales. Proponemos analizar estructuras formales minimalistas a partir de 1953 en México y desarrollos posteriores. Los productores incorporaron conceptos plásticos de los norteamericanos determinando un parangón entre ambas estructuras objetuales. Por último, pretendemos asentar la vigencia del minimalismo local en el arte globalizado con ensamblajes escultóricos. 
Por otro lado, es necesario correlacionar el ensamblaje mexicano como aportación al minimalismo, ya que contiene estructura mínima por su fragmentación de partes industriales unificadas. Además, el neoensamblaje corresponde a "la acción o efecto de unir: ideas, objetos industrialescotidianos, conceptos, materiales, técnicas, medios alternativos que unifiquen en un todo la forma ensamblada" (Estévez, 2012, p. 60). El ensamblaje minimalista es construido por planos, prismas geométricos y en ocasiones con formas orgánicas parcialmente ajustadas a una forma sensible planimétrica. Así también, consideramos al minimal art como fundamento de lo escultórico mexicano al sistematizar la aportación del ensamblaje. El reduccionismo de la tendencia marca una etapa compositiva del productor, con objetos de epidermis mínima y reduce la expresión de forma. El ensamblaje conformado por fragmentos yuxtapuestos e híbridos se forja con implementos industriales como láminas, varillas, soldadura y uniones extremas con otros componentes. Ejemplificamos lo anterior con los ensamblajes de equilibrio de placas de acero realizadas por Richard Serra (San Francisco, 1939), en la obra The Matter of Time (1994-2005) ubicada en el Museo Guggenheim de Bilbao.

En defensa de la génesis del minimal art y su evolución en la producción escultórica local, confirmamos el desarrollo paralelo entre los escultores de ambos países. Las propuestas mexicanas de 1953-1958 son obras públicas como el Museo Experimental El eco y Las Torres de ciudad Satélite, que dieron la pauta para el desarrollo de la vanguardia por los precursores mexicanos. En tal virtud, Goeritz "seems to have anticipated certain ideas of the minimal artists" 2 (Battcock "et al", 1995, p. 19). Además, las obras emblemáticas de carácter minimalista aportaron al espectador la incursión física y libre a los objetos urbanos.

En México necesitamos confeccionar literatura especializada que abone al minimalismo mexicano, en especial su origen escultórico. El auge de la propuesta vanguardista norteamericana de los años sesenta contribuyó a crear un paralelismo en la producción mexicana. Así también, al vincular correspondencias plásticas en la construcción del objeto local a partir de los norteamericanos, contamos con evidencia de estructuras cercanas a la tendencia. Por último, el espectador de obra pública con formato geométrico verificará la cercanía formal-compositiva con el minimal art globalizado. En tal sentido, el veedor participa en las exposiciones de objetos mínimos en nuestros museos y galerías. Por lo que, el objetivo principal en el artículo consiste en dejar constancia de la aportación mexicana en la configuración del minimal art. Además, verificaremos los paralelismos de la producción 
escultórica entre estadounidenses y mexicanos de los años setenta a la fecha. Y por último, demostraremos la vigencia del ensamblaje minimalista en el arte contemporáneo.

\section{Aportación mexicana al minimalismo}

La observación que hace Gregory Battcock, en su libro Minimal Art a Critical Anthology (1968-1995) plantea que la obra de Mathias Goertiz (Danzing, (1915-ciudad de México, 1999), es cercana a las estructuras planimétricas geométricas de la tendencia minimalista. La edificación precursora corresponde al Museo Experimental El eco (1953) considerado la primera obra arquitectónica de carácter minimalista y, además contaba en su interior con la escultura La Serpiente de El eco (1953) forjada con planos laminados que consagra un volumen cerrado a partir de la forma y sistema se sujeción. En tal virtud, la correspondencia escultórica mexicana en el minimalismo es La Serpiente de El eco, que "constituyó toda una innovación en la escultura de la época, por sus formas acentuadamente lineales y fue predecesora del minimal art" (Moyssén, Rodríguez, Acha, Manrique y Del Conde, 1977, p. 70). La obra minimalista de Goeritz y Barragán la apreciamos en su dimensión vanguardista en la Ciudad de México, para abonar sobre nuestro argumento, nos dice Battocock que Goeritz:

...had designed an experimental structure in México City called El eco. The walls and other architectural components of the building were conceived in conjunction with large, Minimal sculptural pieces [La Serpiente de El eco (1953)] that all but took over the interiors [...] In El eco he explored successfully the problem of architectural enclosure (space) and the relationship of Minimal sculpture to the limitations of negative space -the floor, walls, and ceilings. ${ }^{3}$ (1995, p. 19).

Así también, Mathias Goeritz y Luis Barragán, construyeron el conjunto escultórico urbano denominado Las Torres de ciudad Satélite (1957-1958) edificado con prismas triangulares de 37 a 57 metros de altura con cinco módulos de cemento coloreado. La obra pública constituyó un señalamiento urbano para los suburbios de la Ciudad de México y los espectadores la consideran una obra emblemática. Otro antecedente de la génesis del minimal 
art, lo esgrime Víctor Pérez Escolano en su artículo Escalas del minimalismo. Arquitectura y escultura, señala que Luis Barragán es "citado como antecedente minimalista en razón a su elementarismo de matriz popular" (Pérez, "et al." 2003, p. 278).

El siguiente paso minimalista de trascendencia en la obra pública en la Ciudad de México, constituye el Espacio Escultórico (1979) erigida en el campus de la UNAM. La obra monumental de características minimalistas, es construida con 64 módulos poliédricos con base rectangular confeccionados de concreto armado, los objetos de repetición se encuentra alrededor de un cilindro de cemento y piedra de 120 metros de diámetro. La distribución de cada modulo tiene una constante de espacios y volúmenes con libertad de movimiento para el espectador entre cada uno de los prismas.

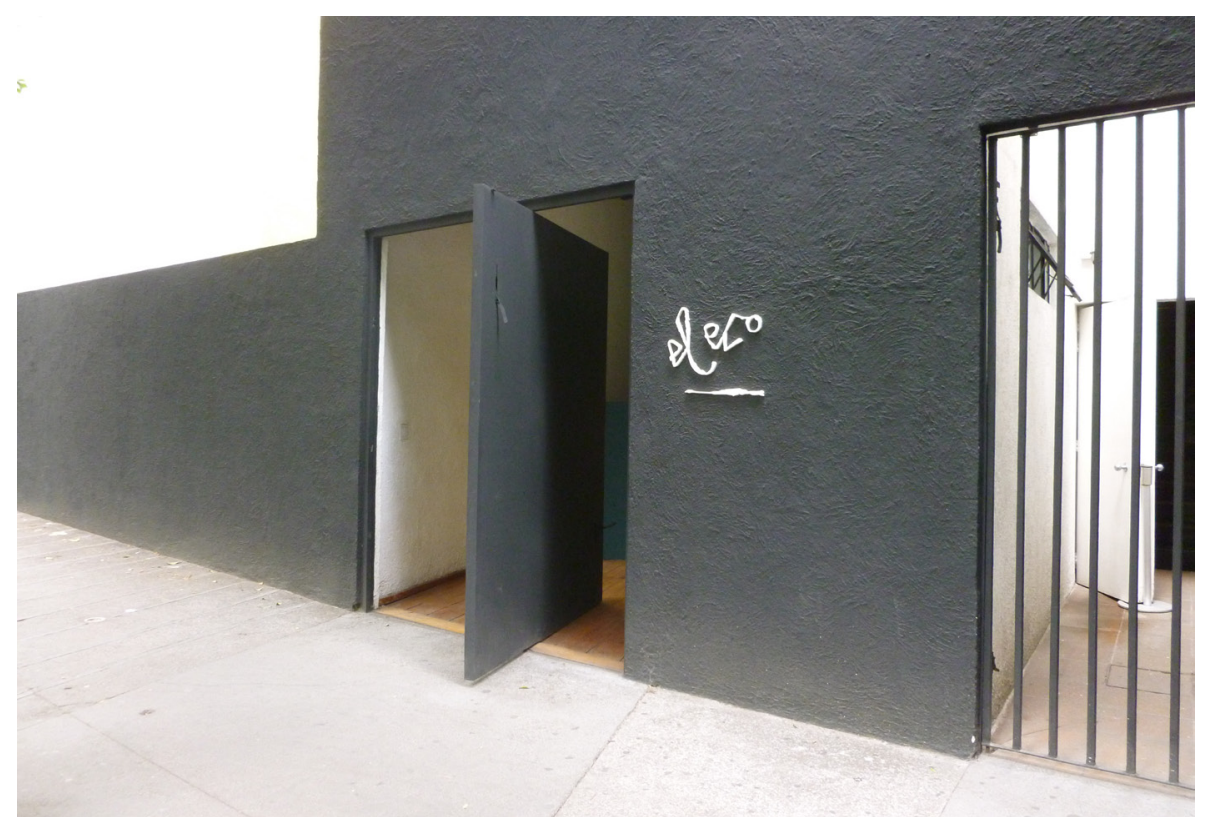

Figura 1. El Museo Experimental El eco (1953), Ciudad de México. Fuente: foto de Pablo Estévez Kubli. (C) 

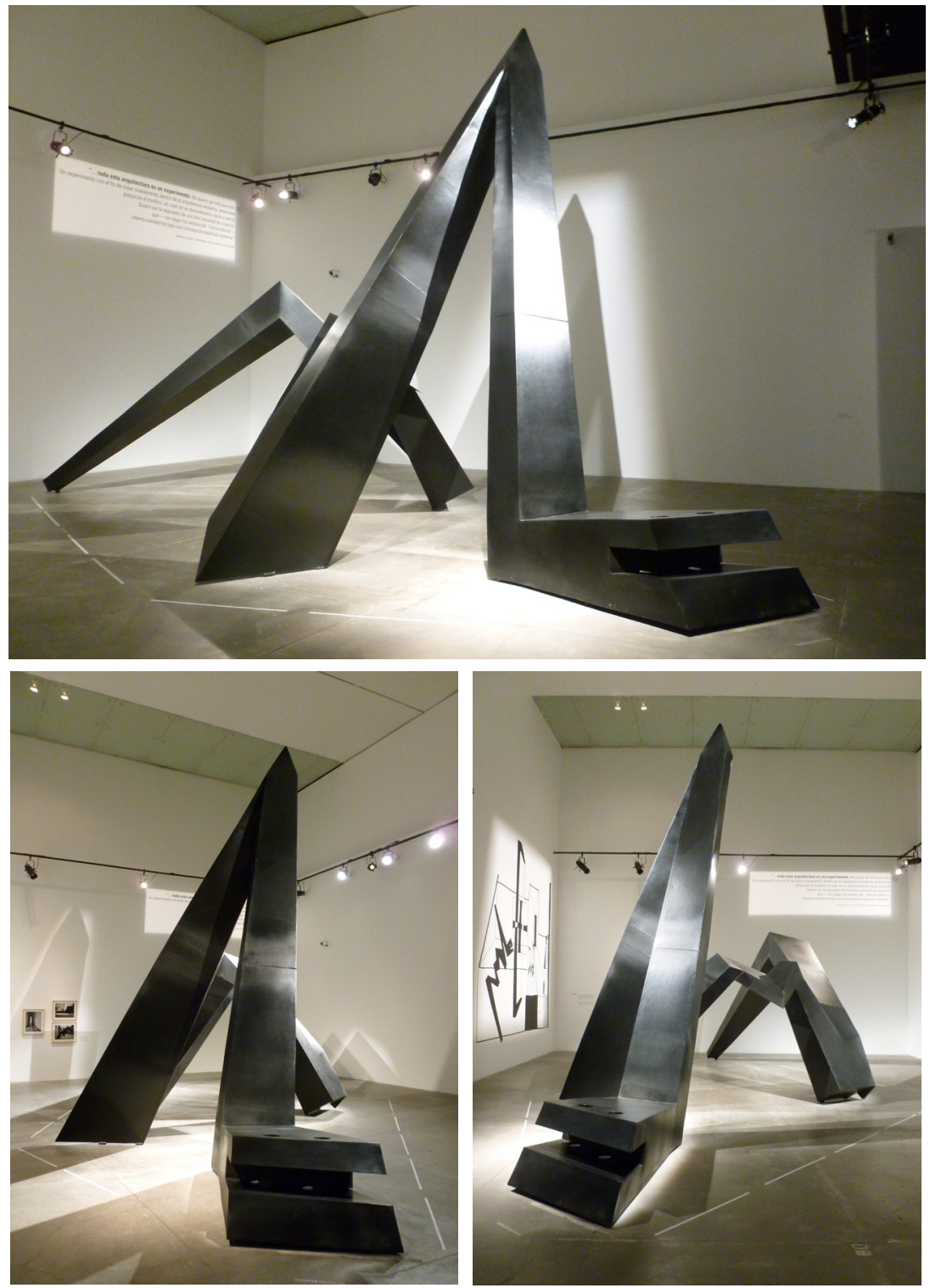

Figuras 2,3,4. La Serpiente de El eco (1953) en la Exposición Desafío a la Estabilidad Procesos Artísticos en México 1952-1967 en el MUAC-UNAM (2014), Ciudad de México. Fuente: foto de Pablo Estévez Kubli. (C) 
Por todo lo anterior, podemos asegurar que el minimalismo nace en México en los años cincuenta con obras trascendentes en la escultura y arquitectura. En Nueva York y en Los Ángeles, California se inicia el minimal art con estructuras geométricas, con procesos industriales de la modernidad y sobre todo con teorías elaboradas por los artistas estadunidenses que sostienen la tendencia. Los productores del minimal art son Robert Morris, Donald Judd, Sol LeWitte, Carl Andre, Richard Serra, Tom Doyle, David Smith y Eva Hesse, entre otros y del lado mexicano contamos con Hersúa, Jesús Mayagoitia, Manuel Felguérez, Sebastián, Gunther Gerszo, Ernesto Hume, Salvador Manzano, Ernesto Álvarez, Pablo Kubli, Ernesto Paulsen, Yvonne Domenge, Francisco Moyao, Xawery Wolski y Thomas Glassford, entre otros.

\section{Argumentación de estructuras minimalistas}

Consideramos dos argumentos que nos condujeron a escribir este artículo para presentar los hallazgos del lenguaje escultórico de artistas mexicanos. El primer argumento corresponde a la obra La Serpiente de El eco (1953) de Goeritz, construida con módulos laminados y remachados, desarrollando la planimetría volumétrica. Las correspondencias de la vanguardia minimalista de estructuras selladas como la obra citada, generaron respuestas directas a prácticas artísticas de autores como Donald Judd, Tony Smith, Sebastián y Jesús Mayagoitia, toda vez, que unificaron criterios plásticos al realizar esculturas geometrizadas de volumen cerrado.

Como segundo argumento, consideramos que la escultura mexicana de mediados del siglo veinte conserva resonancias formales, que aducen al factor espacio, planimetría y temática contextual. La obra minimalista local pertenece a dos variantes; por un lado, estructuras metálicas volumétricas selladas y por la otra, obra planimétrica con vacíos intercalados entre sus partes. El factor planista geométrico de la obra tridimensional (1953-1958) procuró nuestro devenir en el arte actual. Además, verificamos que la aplicación de laminados de diversos materiales provoca un desarrollo pleno en la producción escultórica en ambos países. Por lo que, consideramos que los dos componentes centrales en la ejecución de la obra contemporánea es la planimetría integrada a parámetros minimalistas. 


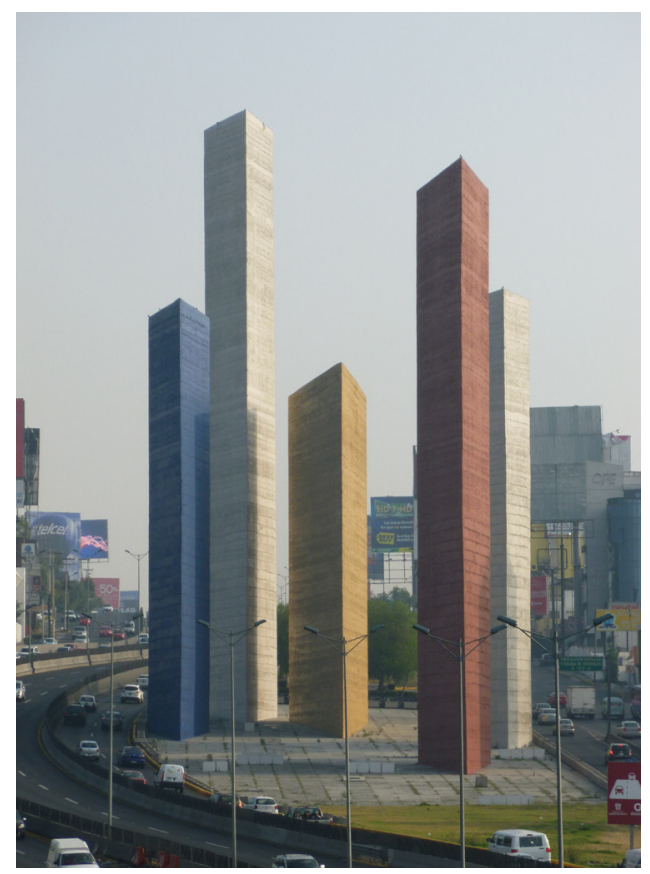

Figura 5. Las Torres de ciudad Satélite (1957-1958) Estado de México. Fuente: foto de Pablo Estévez Kubli. (C)

Así también, el espectador analiza la forma de estructura minimalista descubriendo correspondencias formales, conceptuales y técnicas. Los objetos de la tendencia con la aplicación de insumos de la modernidad maquinista demuestran las equivalencias plásticas entre la producción de nuestros escultores y los estadounidenses. Al analizar las referencias mexicanas del arte minimalista enriquecemos nuestro entendimiento de lo escultórico del minimal. Y nos preguntamos si es pertinente establecer correspondencias en el proceso creativo de los artistas radicados en México con otros fuera del país. En el presente siglo es innecesario cuestionarnos tal circunstancia, debemos aceptar la globalización del arte como un sistema de mutua interconexión. En tal sentido, es procedente que los artistas conserven factores de la tendencia y tengan referencias recíprocas en la producción de objetos de epidermis minimalista. Al demostrar los equivalentes plásticos entre objetos de una misma época, certificamos las influencias directas, parciales o adoptadas por los escultores mexicanos en el desarrollo del minimal art. 


\section{Características del minimalismo en la construcción del objeto}

La fuente sustancial del minimal art corresponde al material industrial consistente en laminados en metal, plástico, madera y aplicaciones tecnológicas en talleres metal-mecánicos. La industria pesada con implementos maquinistas abrió sus fábricas para forjar obras proyectadas por los minimalistas. Los artistas de la tendencia desarrollaron su forma de trabajo en base a la concreción de modelos, bocetos y maquetas a escala. Las obras se realizaron con diversas herramientas del área metal mecánica en procesos de maquinado y forja de laminados. La unión de sus partes fragmentadas de cada objeto derivó del uso intensivo de procesos de soladura, atornillado o remachado, siempre en busca de sistemas para sujetar los fragmentos de la obra. El auge del minimalismo conlleva a una epidermis geométrica volumétrica sellada en productores como Robert Morris, Larry Bell, Ronald Bladen, Tony Smith, Anne Truitt, Judy Chicago, Sol LeWitt, Hersúa, Sebastián y Jesús Mayagoitia.

Otro esquema compositivo en el posminimalismo se genera con planos sueltos con espacios intercalados sin pretender un volumen cerrado. En tal sentido, es conveniente distinguir entre los productores que realizaban estructuras totalmente selladas por sus lados -prismas geométricos- y los artistas que elaboran obras con planos sueltos dejando que intervenga el factor espacio entre sus partes. Los autores planistas crean espacios entre sus partes fragmentadas como Donald Judd, Carl Andre, Eva Hesse, Richard Serra, John McCracken, Manuel Felguérez y Pablo Kubli, entre otros. Los obreros metalúrgicos en general interpretan las proyecciones de los minimalistas creando la obra final con indicaciones precisas en su desarrollo, ya que "suele estar compuesta de formas geométricas únicas o repetidas. Producidas industrialmente o construidas por obreros cualificados siguiendo, las instrucciones del artista" (Meyer, 2011, p. 15).

La característica estadounidense corresponde a la visión conceptual que desarrollaron los artistas minimalistas al proponer eliminar las emociones del espectador sobre sus estructuras, Meyer nos dice que "erradican todo vestigio de emoción o de decisión intuitiva" (2011, p. 15). El esquema anterior es inexacto, ya que emerge algún tipo de sentimiento del veedor sobre la pieza. El proceso creativo que desarrollaron los minimalistas contenía la encomienda de forjar esculturas sin provocar gran emoción, lo que proponían era un cambio radical de la escultura previa a la tendencia y por lo tanto, evitar la apreciación del artefacto de manera tradicional. El cambio de paradigma lo 
describe Edward F. Fry en su artículo Sculpture of the Sixties ${ }^{4}$.

...el arte minimalista era demasiado extremo para encontrar su lugar en la historia del arte, ya que se trataba de una tentativa deliberada para escapar a los estilos anteriores al siglo XX y acabar con los métodos de composición que estaban en la base del arte occidental. (Guasch, 2009, p. 159).

En tal situación, entran a la discusión los factores formales, materiales y emocionales del espectador que provoca el objeto-escultura posminimalista contemporáneo. Otra característica del minimalismo es su estructura corpórea que se adquiere al utilizar sistemas industriales sin aparente emoción por sus laminados, estructura y sistema de conexión mecánica. El objeto minimalista es por esencia un cuerpo mínimo al ser conformado con pocos fragmentos híbridos o yuxtapuestos en su composición. Las estructuras se conciben libres de ataduras $\mathrm{u}$ oropeles en su forma, ya que se conforman con planos lisos y que provocan esquemas de reducción de forma, en tal correspondencia "el arte minimalista utilizó los objetos de producción industrial para lograr una reducción de tipo formalista" (Meyer, 2011, p. 18).

Además, el factor espacio se intercala entre las partes del objeto, es sin duda la característica principal del arte minimalista planimétrico, ya que genera vacíos interiores para establecer la forma definitiva. El factor espacio es acompañado conforme al tiempo, desplazamiento de partes y recorrido visual. El veedor para encontrar la forma definitiva se desplaza al rededor del objeto y logra observar el contenido de la obra en tiempo-espacio. En las estructuras planimétricas minimalistas el factor espacio juega de manera preponderante, ya que los vacíos son volúmenes virtuales que acompañan la estructura en su forma definitiva.

Otros elementos escultóricos minimalistas corresponden a la producción en serie, aplicación del sistema modular en repetición, progresión y la toma de decisión constructiva de volumetría cerrada o abierta por planos laminados; ambas acciones generan formas de carácter reduccionista. En un primer análisis de objetos de ambos países, verificamos apariencias de igualdad en el uso indistinto de los factores compositivos señalados para la construcción del objeto. En tal sentido, el primer objetivo de análisis atañe al sistema de agrupación de módulos construidos por volúmenes planimétricos, líneas engarzadas y conjuntos estructurales como las instalaciones de Robert Morris. 
Los ensamblajes minimalistas contienen las características del assemblage histórico, desde apropiaciones, reciclamiento, paráfrasis, materiales neutros hasta el uso indiscriminado de los parámetros del posminimalismo. La integración en la estructura por objetos encontrados, materiales de desecho, procesos industriales y fragmentos híbridos sustentan la forma física del ensamblado. Los escultores aplican la planimetría en sus assemblage y realizan estructuras compositivas de superficie-relieve, exentas y conjuntos que reflejan un lenguaje personal en la elaboración de cada pieza. Los productores mexicanos y estadunidenses comparten ciertos sistemas compositivos que se generan a partir del factor espacio-tiempo, formato liso, estructuras sin aparente emoción y la aplicación de sistemas industriales en la construcción del objeto por talleres especializados.

El material sustantivo para probar los alcances de la producción minimalista entre escultores norteamericanos y mexicanos es el análisis de estructuras concretas. El contraste, análisis y correspondencias se fincan en el uso de elementos formales en la elaboración del objeto de la tendencia. Ejemplificamos lo anterior con las cajas de pared abiertas de Donald Judd de su obra Untitled (1989) y la caja de zapatos de Gabriel Orozco en Empty Shoebox (1993), la primera es de aluminio con plexiglás y la segunda de cartón, pero cada escultor concibe su concepto sobre el espacio abierto de magnitud diferente. Otra vinculación corresponde a las estructuras de los prismas sellados de Tony Smith y su contraste con las esculturas de Sebastián, Jesús Mayagoitia y Hersúa. La diferencia radica en las actitudes de los escultores norteamericanos, ya que escribieron textos teóricos que sustentan la tendencia como en Specific Object (1965) de Donald Judd y, los de Robert Morris Notes on Sculpture (1966) y Anti-Form (1968); los ensayos nos proporcionan lineamientos formales del minimal art. La trascendencia de la postura norteamericana radica en proponer objetos anti-arte y por el lado mexicano, los productores asumieron sus objetos como escultóricos per se.

El estudio comparativo de objetos-estructuras analizados bajo premisas iguales entre obras plásticas de ambos países, nos da pauta para observar el paralelismo entre productores. Al analizar piezas de los escultores observamos características similares, materiales industriales igualitarios, composiciones rígidas geometrizadas y variaciones en la proporción del anti-arte como los norteamericanos o consagrar su esfuerzo formal escultórico en obras públicas como lo ejercen los mexicanos.

El resultado inicial del artículo versa sobre los hallazgos del minimalismo a nivel global, que corresponde a la arquitectura iniciática del Museo 
Experimental El eco, y a la escultura La Serpiente de El eco emplazada en el interior del Museo, ambas de 1953. Así también, la obra pública Las Torres de ciudad Satélite (1957-1958), construida por Luis Barragán y Mathias Goeritz; son consideradas las tres obras precursoras directas del minimal art. La evidencia en fotografías que se acompañan al cuerpo del ensayo, adquieren relevancia probatoria, además en la literatura del minimalismo se asienta en forma indirecta la hazaña mexicana. En tal sentido, vinculamos el conocimiento sobre las primeras obras públicas minimalistas (1953-1958) que se edificaron en espacios públicos en la Ciudad de México. En cambio la tendencia norteamericana surge de manera preponderante en la década de los sesenta en Nueva York y en Los Ángeles, California.

Iniciamos la discusión con el análisis de las estructuras tipo Cajas (1989) de Donald Judd al contrastarlas con la caja abierta Empty Shoebox (1993) de Gabriel Orozco. Evidenciamos que son objetos con similitud formal. La diferencia radica en su emplazamiento y materiales. Con relación a la obra mínima planimétrica sellada por sus lados como los prismas volumétricos de Robert Morris en Sin Título -L Beams- (1964-1967), al respecto Pérez nos dice que:

...los poliedros simples, como su Columna de 1961 o el conjunto de piezas acostadas, apoyadas o colgantes de la famosa instalación de la Green Gallery de Nueva York de 1964... [Morris generó] objetos cúbicos independientes o formas elementales de sección cuadrada [las propuestas de Morris consisten en un diálogo permanente entre estructuras, espacio, lugar y espectador]. (2003, pp. 271 y 272).

Además, Morris desarrolla la tipología site-specific que corresponde al lugar-espacio de emplazamiento de la estructura mínima, en tal sentido, Maria Hussakowska-Szyszko nos indica que Morris siguen en la actualidad investigando lugares determinados con connotaciones sociales y políticas que le proporcionen un análisis de ubicación significativa para implantar su instalación denominada Labyrinth-fragments (2010) y que:

The concept of site-specific art, developed by him in the 1960s and assuming a comprehensive analysis of the place, its history its social, cultural and political environment, which justifies and anchors the work...[así también Morris implementa] the expansion of Works 
that transcended their status as object, consequently undermining the understanding of a place through its history and spiritual hollowness. ${ }^{5}$ (Hussakowska-Szyszko, 2011, pp. 1-18).

En tal virtud, la realización y emplazamiento de prismas regulares laminados como obra minimalista, son objeto de diversas aplicaciones artísticas igualitarias en autores de ambos países. Las correspondencias formales de Robert Morris en su obra Sin Titulo-L-Beams- (1964-1967) ${ }^{6}$ y las estructuras prismáticas de Tony Smith Scale as Content (1967) y Free Ride (1962) ambas de formato geométrico cerrado; son cercanas a los prismas sellados que realiza Sebastián, Jesús Mayagoitia y Hersúa. Así también, otros elementos de análisis que utilizan los minimalistas norteamericanos y mexicanos radican en la aplicación de una sintética volumetría de forma y, utilización de módulos en repetición o progresión. Los norteamericanos además de forjar sus obras con producción en serie aducen a conceptos personales que reflejan en sus investigaciones teóricas. Los mexicanos consagran sus estructuras con pensamientos propios sin conceptos unitarios en la construcción del objeto, faltando rigor teórico en la estructuración del objeto posminimalista.

Además, las estructuras geométricas desarrolladas en ambos países contienen epidermis igualitaria a la vista del espectador, ya que son formas cerradas, planimétricas y con proporciones formales similares. Sin embargo, de lado mexicano las propuestas públicas de la tendencia son de gran calidad y emplazadas en todo el territorio nacional, conforme a "la estructura del ritmo geométrico, integración formal sobre bases reticulares y énfasis volumétricos [características principales para unificar a los escultores]" (Pérez, 2003, p. 285).

Por otro lado, con relación al factor compositivo silencio en la arquitectura minimalista, lo ejemplificamos con La Casa Luis Barragán (1948) edificada en la Ciudad de México. Las casas-habitación minimalistas de Luis Barragán generan "la fascinación de los escultores y arquitectos del emergente minimalismo por los modelos puristas de esa arquitectura [Mies van der Rohe y Hilberseimer] en la que domina el silencio" (Pérez, 2003, p. 289). Argumentamos que la simplicidad formal y mínima va de la mano con la composición de carácter intrincado, ya que con menos elementos en su construcción se genera una complejidad formal y ejecutiva en la fabricación del objeto minimalista de epidermis simplista pero de complejidad formal y conceptual. Consideramos distinguir entre lo mínimo y lo elemental, Pérez propone la tipología del minimal art de la siguiente forma, "un orden elemental cuya compleja construcción hace del minimalismo una categoría de fortuna contemporánea, con la que una larga mirada a la arquitectura moderna permite 
establecer el substrato histórico de uno de los capítulos más brillantes de la escultura del siglo XX" (2003, p. 289).

Así también, las fotografías de Hiroshi Sugimoto en su serie Seascapes -fotografías de la naturaleza- (1980-2003) nos demuestran la vigencia del minimalismo en otras disciplinas fuera de las estructuras escultóricas. Sugimoto artista japonés traduce la homogeneidad del paisaje retratado en estructuras mínimas bidimensionales con desplazamientos de tonos que van del negro intenso al blanco luminoso y con una variedad de grises por módulos horizontales que construyen la imagen. La utilización del horizonte bajo y alto denota la profundidad total que Sugimoto imprime en sus fotografías de plata-gelatina en un formato estandarizado. Además, la luz y las sombras de las atmósferas paisajistas de la naturaleza generan la estructura del objeto en sus diversas degradaciones de tonos, ya que son fotografías abstractas en las que existen diversas realidades conforme al sistema modular planista. Peter Krieger en su ensayo La estética del mar y otros minimalismos de Hiroshi Sugimoto, nos dice que:

...comunica el espíritu minimalista [...] En la serie se retratan los fenómenos esenciales y enigmáticos de luz, sombra, agua y atmósfera [...] Los paisajes marítimos infinitos, tan atractivos como aterrorizantes para el ser humano, sin duda exigen contemplación, evocan emociones y generan codificaciones. (2010, p. 137).

Otro referente comparativo entre mexicanos y norteamericanos, lo relacionamos a la serie fotográfica de secuencia marítima de Sugimoto con las pinturas de Mark Rothko (Lituania, 1903-Nueva York, 1970) que reflejan la inmensidad de las planicies, desiertos y montañas; provocando en el lienzo la unificación de tierra-cielo. En la obra Rojo, blanco y marrón de 1957 y en Sin titulo (mural de Seagram) de 1959, ambas de Rothko visualizamos el factor espacio en las composiciones que proyectan amplias zonas rectangulares de color. Los planos de pigmento son módulos que generan ritmos de color y forma mínima de carácter difuso. Rothko en sus años de formación en el estado de Oregón se acerca al minimalismo formal con franjas planistas que derivan del paisaje norteamericano de extensión colosal. Ante las obras de Sugimoto y Rothko, empequeñecemos ante el espacio-tiempo que representa, ya sea en fotografías de la naturaleza como en la serie Seascapes -mar-cieloo en las pinturas de Rothko con vacíos de tierra-cielo que corresponden a ambientes brumosos. 
Proponemos para ejemplificar el alcance del posminimalista la obra TorresDivisiones (1990-2013) de Pablo Kubli. Es un ensamblaje industrial realizado con dos elementos iguales en tamaño y proporción, enfrentados al factor espacio-tiempo entre estructuras y, transitable en su interior por el espectador. Los dos módulos de seriación igualitaria provocan vacíos entre las láminas de aluminio atornilladas. Las sombras y luces que emite la estructura metálica se transforman al ser intervenidas físicamente por el recorrido del espectador al sucumbir entre los módulos. La repetición de planos rectangulares genera armonía y solidez en su estructura de repetición, simultaneidad y se crean ritmos interiores entre cada módulo. Además, las líneas de las estructuras que conectan los módulos son tornillos amplios que unen los planos verticales igualitarios en forma y conexión, creando vacíos interiores entre cada fragmento. El veedor experimenta en su recorrido físico estructuras con características minimalistas.

Concluimos que la aportación mexicana es contundente, ya que los productores desarrollaron esculturas de formato minimalista proponiendo características formales para la tendencia. La constancia es determinante con obras urbanas a partir de los años cincuenta del siglo pasado como La Serpiente de El eco (1953), construida por laminados metálicos en formato geometrizante. Las correspondencias plásticas entre mexicanos y estadounidenses las visualizamos conforme a los prismas planimétricos de Tony Smit, Donald Judd y Robert Morris en contrapunto con esculturas de Sebastián, Jesús Mayagoitia y Hersúa. Además, confluye en la escena mexicana el ensamblaje escultórico con raíz minimalista de Pablo Kubli, ya que sus obras contienen yuxtaposición e hibridación de materiales que unifican fragmentos industriales en artefactos de corte minimalista. En tal sentido, demostramos con evidencia que la génesis del minimal art se produjo en la Ciudad de México con obra pública y, que la línea de investigación sigue vigente con propuestas contemporáneas. Además, necesitamos desarrollar literatura mexicana para analizar los procesos creativos de los escultores nacionales y su correlación con los estadounidenses. Las esculturas abordadas como evidencia conservan un juego espacial por movimiento, planos, líneas de conexión y sombras entre fragmentos con emplazamientos en lugares específicos. Por lo que, debemos construir la tipología del minimalismo mexicano en su devenir histórico contemporáneo. 


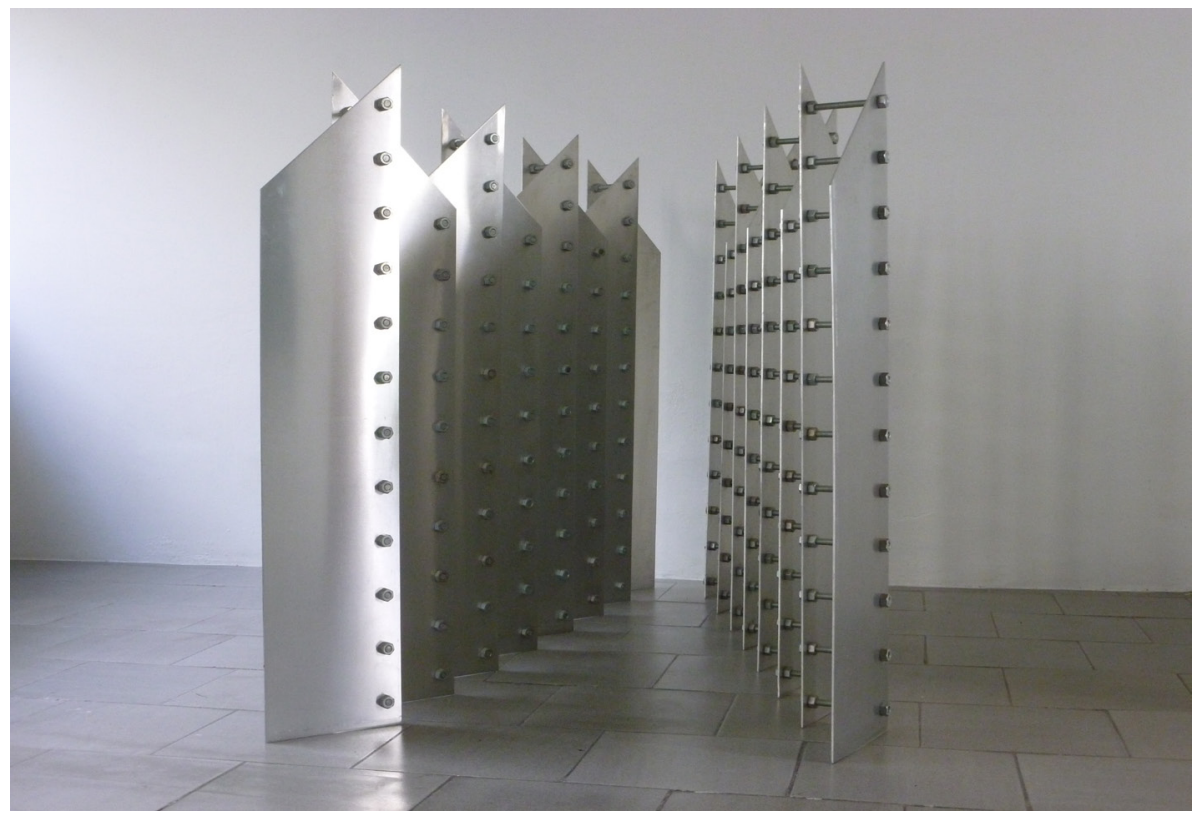

Figura 6. Pablo Kubli, Torres-Divisiones (1990-2013). Aluminio, tuercas y tornillos. 125 x 200 x $45 \mathrm{~cm}$ cada uno. Colección del autor. Fuente: foto de Pablo Estévez Kubli. (C)
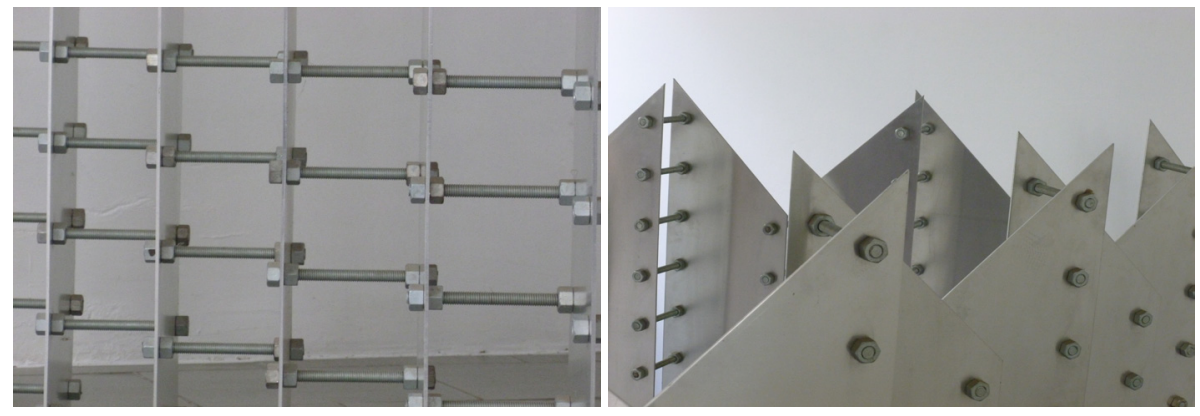

Figuras 7, 8. Pablo Kubli, Acercamiento de la obra Torres-Divisiones (1990-2013). Colección del autor. Fuente: foto de Pablo Estévez Kubli. (C) 


\section{Notas}

${ }^{1}$ Rose, B. (1965, octubre-noviembre). ABC Art. Art in America, 57-69.

${ }^{2}$ En tal virtud Goeritz parece haber anticipado ciertas ideas del minimalismo. [predijo los parámetros minimalistas como los factores de composición, materiales y sobre todo la aplicación del elemento espacio tanto en la escultura como en la arquitectura]. La traducción es nuestra.

${ }^{3}$ Mathias Goeritz había diseñado una estructura experimental en la Ciudad de México llamada Museo Experimental El eco. Las paredes y otros componentes arquitectónicos del edificio fueron concebidos en colaboración con grandes piezas escultóricas del Minimal [La Serpiente de El eco (1953)] todo se encontraba en los interiores [...] En El eco exploró con éxito el problema de la caja arquitectónica (espacio) y la relación de la escultura Minimal con limitaciones de espacio negativo, -el piso, las paredes y techos-. La traducción es nuestra. ${ }^{4}$ Fry, E. F. (1967, septiembre-octubre). Sculpture of the Sixties, Art in America, 28.

${ }^{5}$ El concepto de arte de sitio especifico, desarrollado por [Morris] en la década de 1960; supone un análisis exhaustivo del lugar, su historia, su entorno social, cultural y político, que justifica y ancla su obra [asi también desarrolla] la expansión de las obras que trascendieron su condición de objeto, por consiguiente, socava la comprensión de un lugar a través de su historia y su vacuidad espiritual. La traducción es nuestra.

${ }^{6}$ En la exposición Primary Structures: Younger American and British Sculptors en el Jewish Museum, New York (1966), Robert Morris presentó sus estructuras prismáticas minimalistas.

\section{Referencias}

Battcock, G., Wagner, Alloway, Bochner, Bourdon, Calas, Fried, Glaser, Goossen, Graham, Greenberg, Hutchinson, Lee, Lippard, Morris, Mussman, O'Doherty, Perreault, Rainer, Rose, Rosenberg, Sandler, Sharp, Varian, Wagstaff, Wollheim y Raysse, (1995). Minimal Art a Critical Anthology. Battcock (Ed.). Los Angeles California: University of California Press.

Estévez, P. J. (2012). El Ensamblaje Escultórico: análisis y tipologías objetuales del Arte Contemporáneo Mexicano. México: UNAM.

Guasch, A. M. (2009). El Arte del Siglo XX en sus Exposiciones. 1945-2007. España: Ediciones del Serbal.

Hussakowska-Szyszko, M., (2011). Labyrinth-fragments. Robert Morris and his installation for ms Lodz 2010. En RIHA Journal 0032 (15 November 2011), URN, URL, pp. 1-18. Recuperado 20 de Octubre de 2013, de: http://www.riha-journal.org/articles/2011/2011-oct-dec/hussakowskaszyszko-labyrinth-fragments 20.10.2013 
Krieger, P., Hernández, García, Dallal, Jácome, Argan, Volkow, Fernández,

Noelle y Hendrich (2010). La estética del mar y otros minimalismos de Hiroshi Sugimoto. México: Anales del Instituto de Investigaciones Estéticas, Número 96, UNAM, pp.133-144.

Moyssén, X., Rodríguez, Acha, Manrique y Del Conde (1977). El

Geometrismo Mexicano. México: UNAM, Instituto de Investigaciones Estéticas.

Meyer, J. (2011). Arte Minimalista. Londres: Phaidon Press Limited. Pérez Escolano, V., Rodríguez, LaHuerta, Bonzal, González, Llorens,

Castro, Hernández, Navarro, Marchán, De Barañano, Jiménez, Nieto y Arnaldo. (2003). Escalas del minimalismo. Arquitectura y escultura. En ¿Qué es la escultura moderna? Del objeto a la arquitectura. Martín de Argila (Ed.). Madrid: Fundación Cultural MAPFRE VIDA.

Schneckenburger, M., Ruhrberg, Frickey y Honnef (2001). Minimalistas norteamericanos y europeos. En Arte del Siglo XX. Walter I. (Ed.). Madrid: Taschen.

Pablo Estévez Kubli: Profesor en la FAD-UNAM. Universidad Nacional Autónoma de México, Facultad de Artes y Diseño.

Contact Address: Av. Constitución No 600, Col: La Concha, C.P. 16210 , Delegación Xochimilco, México Distrito Federal (Facultad de Artes y Diseño) México.

E-mail address: pablokubli@yahoo.com.mx 